infiltration of the cornea. In the metastatic cases recorded there has been a localized focus of sepsis in some other part of the body.

I believe that the condition is not rare in severe septicaemic plague. Recently I saw ring abscess well marked in both corneae of a female plague patient. When I first saw her she was lying semi-conscious on the fourteenth day of the disease. The corneal infiltration was then definite, but it was more so, and yellowish in tint, two days later. She died on the seventeenth day. There was no evidence of suppuration elsewhere; the only bubo was a moderately enlarged gland, the size of an almond, in the left groin.

In Bombay, years ago, I was struck with the very marked shrinkage of the flattened cornea, down to as little as $3 \mathrm{~mm}$., in atrophic eyeballs from plague endophthalmitis, seen at various intervals after recovery from the disease. Possibly, in these there may have been annular infiltration, which had resolved.

Yours, etc.,

H. HeRBERT, Lt.-Colonel, I.M.S.

\title{
A NEW FORM OF BIFOCAL LENS
}

To the Editor of The British Journal of Ophthalmology.

SIR,-Mr. Bardsley's ingenious scheme for solving the problem of the inclination of the lower segment of a bifocal combination is only of use for the limited case of those patients who are myopes and able to afford the very costly glass described by him. I wish to suggest that the problem may be attacked in another way. Percival, in his book, "The Prescribing of Spectacles," gives, on page 133, the necessary formulae for calculating the cylindrical effect of tilting a lens. A table might be drawn up from this showing the effect of tilting lenses of various strengths to an average reading inclination of $25^{\circ}$. All the surgeon would now require to do would be to refer to the table and prescribe a cylinder for the lower segment of the bifocal sufficient to neutralize the prismatic effect produced by the tilting. This method would be applicable to convex and concave lenses. I hope Mr. Bardsley, with the help of Dr. Fison, may consider the possibility of working out such a table. I must confess that I cannot trust my own mathematical talents to deal with the task.

I am, Sir, yours faithfully,

E. ERskine Henderson. 